\title{
Using XEVA video sensors in acquiring spectral reflectance coefficients
}

\author{
Piotr Walczykowski, Agnieszka Jenerowicz, Agata Orych \\ Military University of Technology, 2 Kaliskiego st., Warsaw 00-908, Poland
}

\begin{abstract}
The rapid and accurate detection and identification of water pollutants play an enormous role is preserving water ecosystems and protecting the surrounding environment. Such detection and identification are usually conducted in situ, using traditional measurement methods. These methods however are very time consuming and costly especially when conducting them on a large scale. A team of specialists from the Department of Remote Sensing and Photogrammetry from the Military University of Technology in Warsaw have been taking part in a project entitled "IRAMSWater - Innovative remote sensing system for the monitoring of pollutants in rivers, offshore waters and flooded areas" (PBS1/B9/8/2012) financed by the polish National Centre for Research and Development. Its main aim is the creation of a remote sensing system based on hyperspectral sensors which will enable the evaluation, detection and distribution of biological, physical and chemical pollutants in the examined waters in real time. These analyses will be conducted based on spectral characteristics of a wide selection of pollutants. In order to acquire these spectral characteristics in a precise manner from hyperspectral data, the research team was required to establish a precise methodology for obtaining these data. The most common approach is to acquire the imagery first, ensuring that each scene contains at least one reference panel with a well know spectral characteristic, and then transforming the imagery and calculating the spectral response curves during post-processing. The IRAMSWater team had proposed a method of extracting precise reflectance coefficients in quasi real time without the need for using a reference panel on the scene. This is done by precisely determining the camera exposure parameters in laboratory conditions. The article presents laboratory experiments conducted using two XEVA video sensors (400-1700 nm spectral data registration), which allowed the authors to form a methodology for acquiring spectral reflectance coefficients of objects within a scene without the need for a reference panel.
\end{abstract}

Keywords: spectral reflectance coefficients; XEVA video sensors; IRAMSWater; reference panel; exposure parameters.

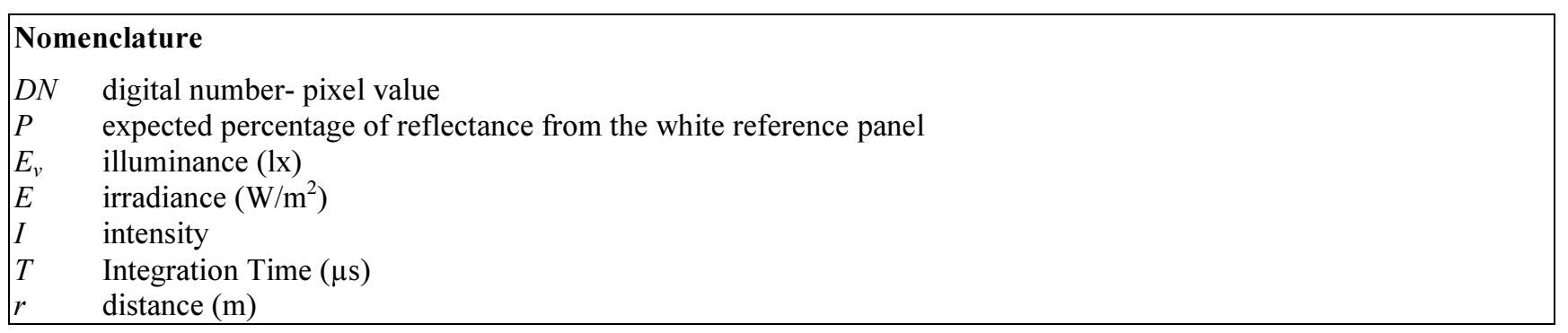

\section{Spectrometry}

Modern remote sensing allows to obtain a number of very detailed information about investigated phenomena. The significant development of technology, especially of sensors, methodologies and electronics, that enabled construction of more sensitive and precise measurement devices, is the driving force behind progress in remote sensing nowadays.

Spectroscopy as the foundation of modern remote sensing, is a science that is dealing with recording and interpretation of electromagnetic spectra. Those spectra are the results of interactions between electromagnetic radiation with matter of different properties. Spectroscopy is used for all issues related to the description of matters' structure, plasma diagnostics or identification of substances and is an object of studies in the field of physic, astronomy, chemistry, genetic engineering, natural science and many others [1].

Optical spectroscopy is one of a number of spectroscopic techniques. It is the study of the interaction between light and matter. Thereby spectrometric measurements are measurements of photons in the function of wavelength. Because the

Corresponding author: Agnieszka Jenerowicz. E-mail address: jenerowicz_a@yahoo.com

http://dx.doi.org/10.3846/enviro.2014.068

(C) 2014 The Authors. Published by VGTU Press. This is an open-access article distributed under the terms of the Creative Commons Attribution License, which permits unrestricted use, distribution, and reproduction in any medium, provided the original author and source are credited. 
reflected and absorbed radiation don't change the frequency of the radiations' monochromatic components and because all particular particles absorb radiation in a characteristic way (depending on wavelength used and the chemical bonds making up the investigated substance) [2], it is possible to identify objects and substances using just their spectral characteristics.

\subsection{Spectral reflectance coefficients}

Each substance reflects a different amount of radiation and with a change in wavelength the value of this varies. Spectral reflectance coefficients represented in a function of wavelength are commonly known as "spectral characteristic" describes a curve presented in the Cartesian coordinate system, which is a graphical representation of the dependence of the reflectance coefficients of an object's surface from the wavelength of the incident radiation. These characteristics allow for the identification of the vast majority of objects and are stored in a spectral libraries. Moreover, each substance has certain distinct bands of the electromagnetic spectrum in which an analysis of the reflectance allows for the assessment of the quality or condition of the tested objects. By analyzing spectral reflectance coefficients in different spectral bands is possible to distinguish substances which seem to be very much alike. Knowledge of the shape of these curves can help us in the selection of spectral channels to acquire in order to detect an object or in a classification process to highlight features or whole objects that are being analyzed. Such analyses of spectral characteristics are used in a project entitled "IRAMSWater - Innovative remote sensing system for the monitoring of pollutants in rivers, offshore waters and flooded areas" (PBS1/B9/8/2012) financed by the polish National Centre for Research and Development, conducted by a team of specialists from the Department of Remote Sensing and Photogrammetry from the Military University of Technology in Warsaw, to evaluation, detection and distribution of biological, physical and chemical pollutants in waters in real time. All analyses will be conducted based on spectral characteristics of a wide selection of pollutants.

\subsection{Acquiring spectral reflectance coefficients - traditional methods}

For direct measurements of spectral reflectance coefficients of objects, an optical measuring device known as a spectrometer is used. The spectrometer is a device used for measuring and analyzing light. Depending on the type of light that is used to illuminate an investigated sample and on the method of it registering, we can distinguish two types of spectrometers: spectrophotometers and spectroradiometers. In both cases, the most important element is the dispersion element (prism or diffraction grating), which splits light into its components. Beam passing through a prism is diffracted by an angle $\delta$, which depends on the refractive index of the prism. Light of different wavelengths is deflected at different angles. In the case of spectrophotometers, the radiation beam from the light source of the instrument is split into very narrow ranges. Next the radiation is directed to the sample. Using transmission spectrophotometers, the amount of light that passes through the sample is tested and with the reflective instruments- the amount of radiation reflected from the material is measured. In the case of spectroradiometers, an independent radiation source illuminates the sample and all the reflected radiation is collected and then split by the prism. Then the amount of split radiation in every narrow wavelength range is read out [1], [3].

Spectral reflectance coefficients obtained with spectroradiometers and spectrophotometers are characterized by a high accuracy. However, due to the nature and structure of the instruments used for this purpose, spectral characteristics can be obtained only from one point of the investigated object at any given time, what is extremely problematic when the structure of the investigated object is not homogenous and is changing in time. Moreover, high quality spectrometers are only available for in situ (from close range) and laboratory measurements, and there is lack of variety of aerial spectrometers [4-5].

\subsection{Acquiring spectral reflectance coefficients - imaging methods}

In order to obtain spectral characteristics of an investigated object using a reflection imaging sensor, it is necessary to use, along with sensor, suitable filters - traditional inference filters or tunable electrooptical filters. The spectral reflectance coefficients are determined on the basis of the calculation of the ratio of the digital number value (DN) of the investigated object in every electromagnetic spectrum range and the DN of a reference sample with a known reflectance value in each spectral range.

In literature we can find a lot of examples of determining spectral characteristics from satellite images (i.e. Landsat, CHRIS/ PROBA and MERIS, etc.) and simultaneous in situ spectral measurements of water, vegetation, etc. (with spectroradiometers and spectrophotometers) [6-8]. As the reference panel white [9-10] reference panels are also used, enabling calculation of spectral characteristics.

According to our concept it will be possible to obtain spectral reflectance coefficients during laboratory and in situ measurements without using reference data, only with the use of an imaging camera. The basis for obtaining the correct reflected values in each registered channel of electromagnetic radiation is the proper registration of images, which will be ensured by correct exposure parameters. The aim of this paper is to present the relationship between the variables that can occur in the registered scene (the amount of light and the distance from the target), and the sensors two exposure parameters - aperture and exposure time. The result should be a developing a formula for establishing the optimal exposure parameters recorded at the selected scene. One of the expected advantages of this approach will be possibility of imaging in different 
conditions without using reference panels, which will be extremely useful when acquiring images from an UAV (Unmanned Aerial Vehicle).

\section{Development of methodology for determining exposure parameters of XEVA cameras}

The aim of this article is to describe methodology developed for determining the exposure parameters of two XEVA cameras.

In order to perform the task, an empirical approach was selected based on a series of measurements performed under laboratory conditions. The purpose of the measurements was to analyze the impact of the following parameters on the value of the pixel brightness- digital number (DN) of a white reference panel:

- utilization of the cameras calibration files,

- distances between camera and the white reference panel,

- quantities of light detected by the detector (aperture value, irradiance and exposure time)

\subsection{XEVA Video sensors}

For the study two different XEVA video cameras were used, both produced by XENICS and both play import ant role in IRAMSWater project.

One of used cameras is a thermostabilized camera XEVA 1.7- 320, model XEVA- 4246. XEVA 1.7- 320 is a high quality digital video camera, with a thermo-electrically cooled InGaAs detector head which registers spectral information in the 900-1700 $\mathrm{nm}$ range. This camera has a wide range of possible applications, such as: image acquisition in the visible and infrared electromagnetic spectrum range, hyperspectral imaging, semiconductor inspection and high temperature thermography $\left(300^{\circ} \mathrm{C}\right.$ to $1200^{\circ} \mathrm{C}$ range or up to $\left.2000^{\circ} \mathrm{C}\right)$. [11]

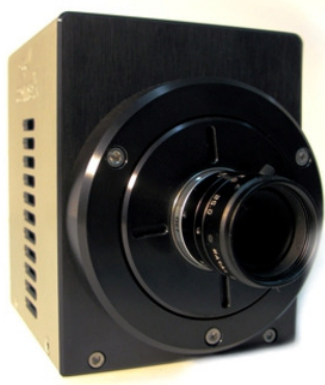

(a)



(b)

Fig. 1. Cameras: (a) XEVA 1.7-320 TE1; (b) XS 1.7-320

The second camera is a compact XS-1.7-320. Like XEVA 1.7- 320 it allows to obtain images in visible and infrared electromagnetic spectrum range. The XS-1.7-320 unit has an InGaAs detector array which is sensitive up to $1.7 \mu \mathrm{m}$. Unlike XEVA, the XS camera does not have thermo-electrical cooling of the detector [11-12].

Both cameras have $16 \mathrm{~mm}$ lenses. Moreover both are small (XEVA 1.7- 320 : 90×110×110 mm; XS-1.7-320: $50 \times 50 \times 50 \mathrm{~mm}$ ) and light (both up to $1 \mathrm{~kg}$ ), what is a big advantage when using them in different conditions: both in laboratory and outdoors.

\subsection{Experiment set-up}

To ensure the accuracy and repeatability of measurements, a number of assumptions about the measurement methods and measurement station were made, such as:

- Measurements were performed in a totally darkened lab, which provided full control over the light conditions in laboratory.

- All calculations were done on DN values of exactly the same surface area of a white reference standard - the Zenith Lite SG3151. It is a $200 \times 200 \times 11 \mathrm{~mm}$ reference panel which has a $95 \%$ lambertian reflectance in $250-2500 \mathrm{~nm}$ range.

- A constant and reliable light source was used throughout the experiment - ASD Inc Pro Lamps. These lamps have a $70 \mathrm{~W}$ power and give constant light in the 350-2500 $\mathrm{nm}$ range. Three lamps were used to ensure the studied reference panel was evenly illuminated from all sides.

- Because light intensity (lux) is only measured in the visible range of the electromagnetic spectrum (400-700 nm) and our studied sensors have a sensitivity exceeding that range $(900-170 \mathrm{~nm})$, instead of measuring light intensity we measured light irradiance.

- High accuracy was provided by measuring the irradiance of the light radiation using a Spectroradiometer Field Spec4 instrument with the ASD Remote Cosine Receptor (RCR) placed on the extension of the cameras optical probe, located 
directly behind the measured white reference panel. The RCR was set on a specially prepared tripod to ensure that all conducted measurements of irradiance were done always from the same place and in the same way.

- All measurements were conducted through a specially designed screen (a curtain), whose main purpose was to eliminate the negative effect of backscattering from the light sources. This ensured that the sensor was only registering energy which had been reflected off the white reference panel. In order to eliminate diffraction, the opening in the screen could not be too small relative to the planned experiment set-up geometry. Taking into account the size of the white reference panel, a $130 \times 130 \mathrm{~mm}$ opening was used. Taking into account the restraints of the laboratory and only being able to conduct measurements from max $10 \mathrm{~m}$, such an opening does not cause visible diffractions of the light beams passing through it. This problem can be further neglected due to the fact that DN measurements were taken from an $70 \times 70 \mathrm{~mm}$ area on the white reference panel, which is much smaller than the chosen opening in the screen.

- The experiment set-ups geometry had to be had to be controlled and stable. This was done by ensuring that the surface of the white reference panel was parallel to the surface of the sensors detector. Additionally it was made sure that the optical axis of the cameras always ran through the centre of the detector array, the centre of the screen opening, the centre of the white reference panel, and the centre of the FieldSpec 4 RCR probe.

A diagram of the experiment set-up is shown in Figure 2.

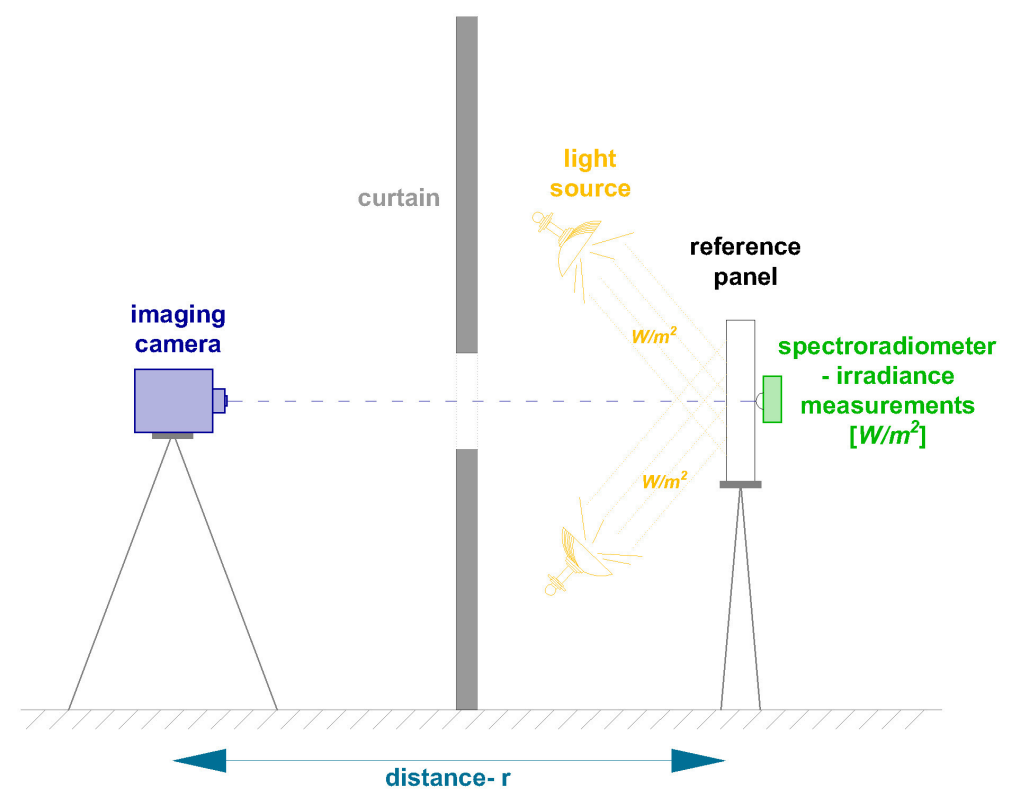

Fig. 2. Measurement station

\section{Results of conducted experiments}

\subsection{Distance from white reference panel}

According to Inverse Square Law the intensity of light observed from a source of constant intrinsic luminosity falls off as the square of the distance from the object:

$$
E_{v}=\frac{I}{r^{2}}
$$

where:

$E_{v}$ - illuminance,

$I$ - pointance (intensity)

$r$ - distance from the light source. [13]

It means that an object that is twice the distance from a point source of light will receive a quarter of the illumination, Figure 3: 


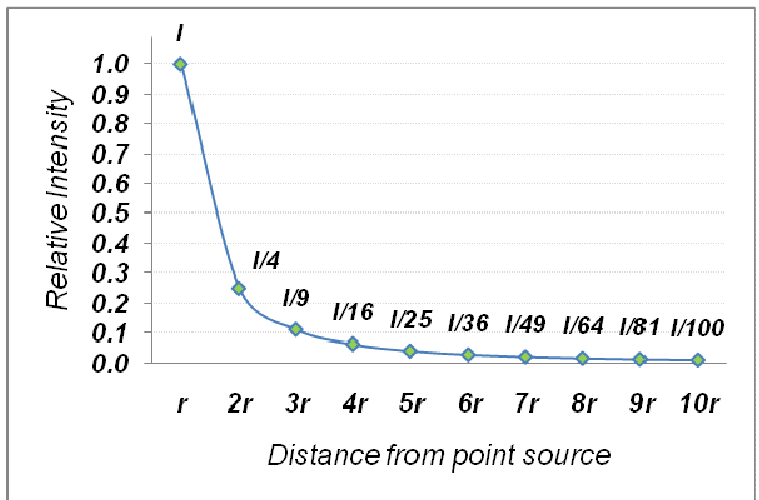

Fig. 3. Inverse square law

However according to the conducted empirical experiments, the distance between the video camera and illuminated white reference panel does not have any influence on pixel values on the image, Figure 4.

In this case Inverse Square Law does not affect the exposure. Along with an increase in distance, the same photons spread out to cover a larger area and are diluted by distance. However with the camera greater distance, the illuminated area also appears smaller, so it balances out. When ten times more distant, the image dimensions are only $1 / 10$ of the original size, which is $1 / 10 \times 1 / 10=1 / 100$ the area. Inverse square law says the light is $1 / 100$ as bright at 10 times distance. So $1 / 100$ the light in $1 / 100$ the area is the same apparent intensity, per unit of area. Summing up, regardless of distance, the camera need to use the same exposure (integration) time.

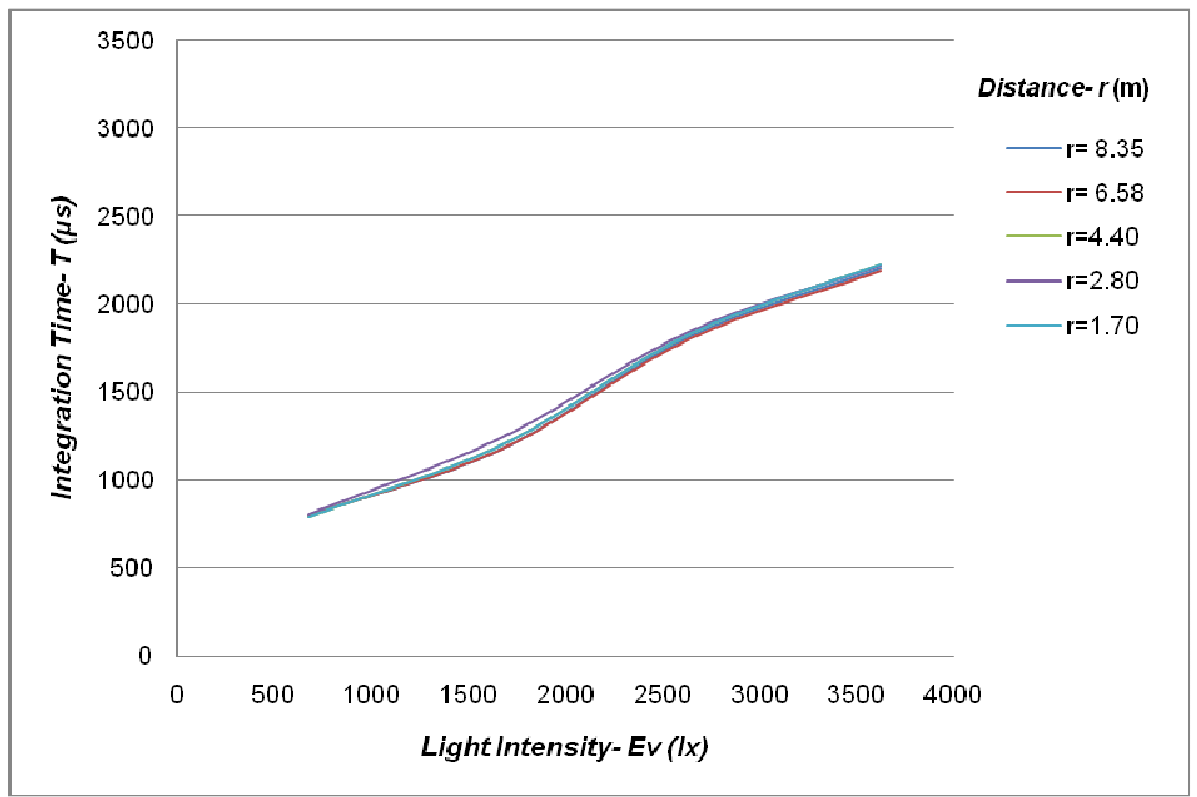

Fig. 4. Relationship between different Integration Times and Light Intensity-results from various distances- $r(\mathrm{~m})$ for XEVA 1.7- 320

\subsection{The Mount of light detected by detector (light irradiance and time exposure)}

In theory of photography and optics, along with a double increase of the exposure time, the radiation that is reaching the photosensitive element (the detector) also increases two-fold, so the pixel value should increase by the same factor until full saturation. The same relationship can be seen in the case of increasing light irradiance on the scene. In the case of thte analog-to-digital converter used in the test camera, to provide a greater dynamic range of the sensor, this relationship is expressed a a linear function. The aim was to determine this relationship by taking many measurements.

All experiments for both cameras were conducted in different lighting conditions (at different light irradiances) and with different exposure times (between 100 and $10000 \mu$ s), see Figure 5 a, b. 


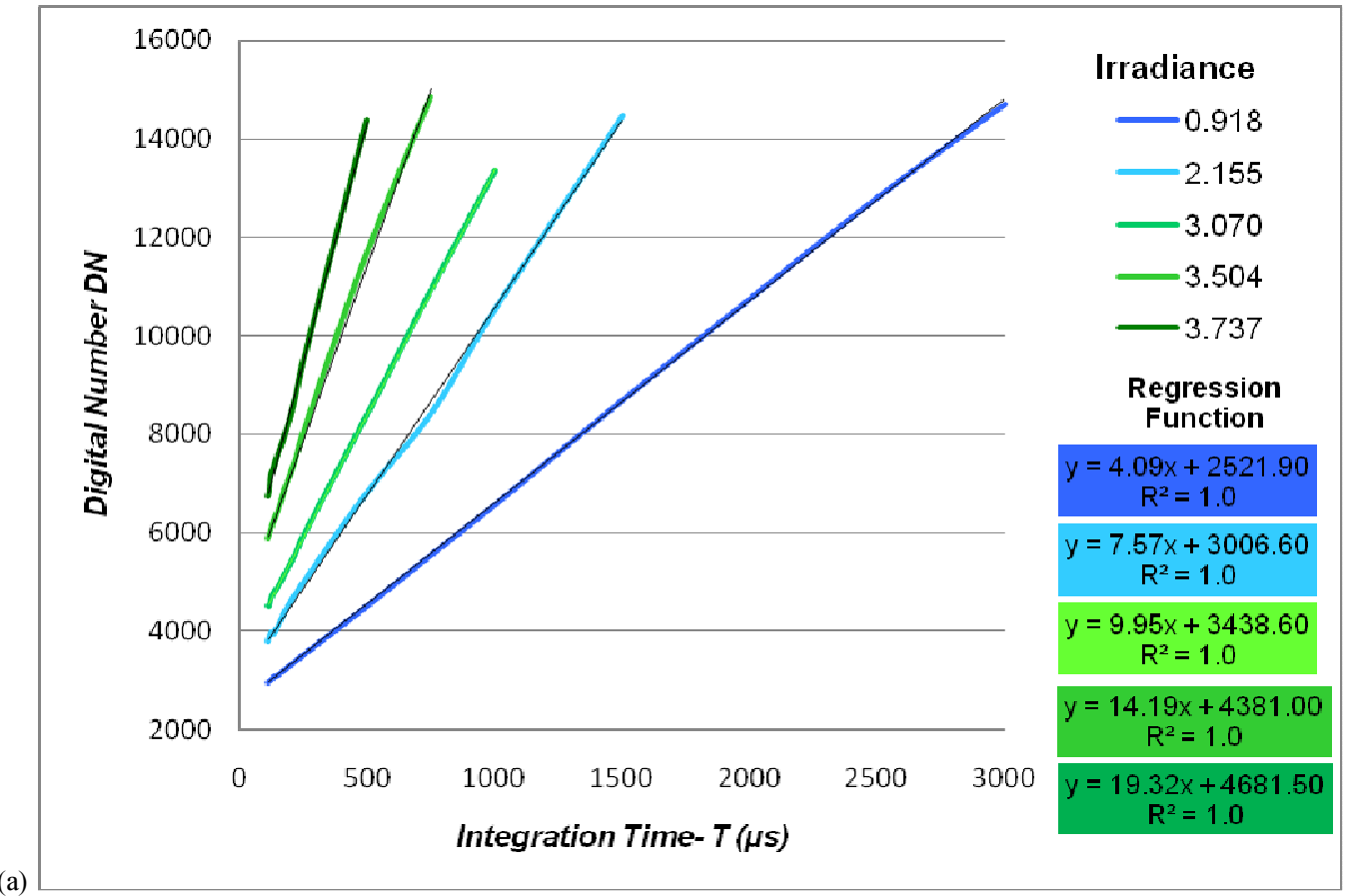

(a)

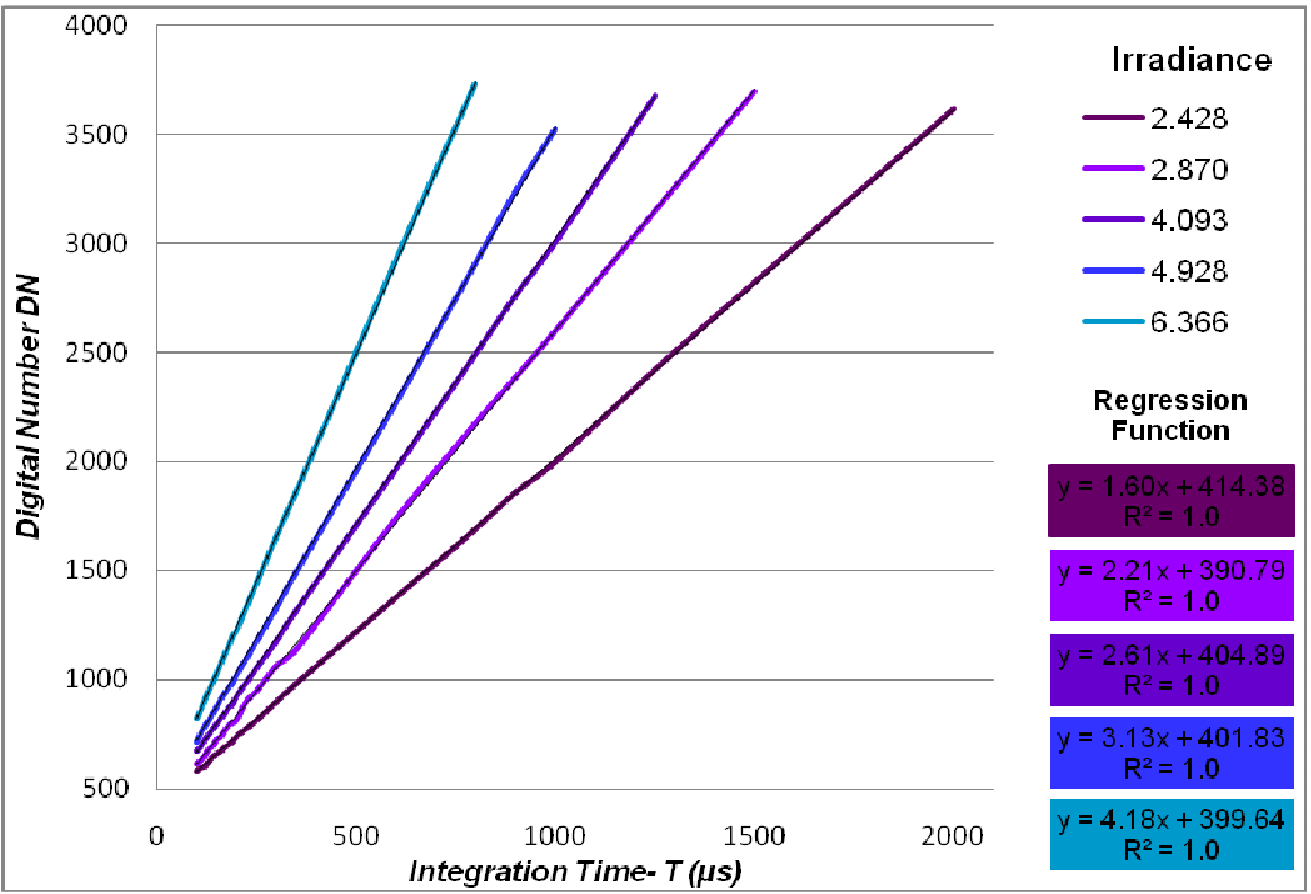

Fig. 5. The relationship between pixel value $(D N)$ and Integration Time for different irradiations- examples; For: (a) XS-1.7-320; (b) XEVA 1.7- 320

Based on the above graphs (Fig. 5 (a) and (b)) it can be seen that the relationship between the irradiance and the pixel value (DN) is a linear one. The factors of the function: directional factor- $a$ and intercept- $b$ are dependent on the exposure time.

From the presented graphs and fixed regression functions approximating relations between $D N$ and $T$, it was possible to determine the function defining the relationship between pixel value $(D N)$, exposure (integration) time $(T)$ and light irradiance $(E)$. Exposure time is calculated from formulas (3) for XS-1.7-320 and (4) for XEVA 1.7-320, only with specific cameras settings (constant gain factors and f-stop set to 16):

$$
\begin{gathered}
T=\frac{\frac{2^{14} P}{100}-471.44 E-2048.1}{2.4293 E+2.2923}, \text { with } D N=\frac{2^{14} P}{100} \\
T=\frac{\frac{2^{12} P}{100}+1.8909 E-411.47}{0.6671 E-0.1121} \text { with } D N=\frac{2^{12} P}{100}
\end{gathered}
$$


where:

$T$ - Integration Time $(\mu \mathrm{s})$

$D N$ - Digital Number- pixel value

$E$ - irradiance $\left(\mathrm{W} / \mathrm{m}^{2}\right)$

$P$ - expected percentage of reflectance from the white reference panel

The validity of the determined formulas has been tested with independent measurements - all results were within $\pm 3 \%$ accuracy.

\section{Summary}

Presented formulas allow for determining the Integration Time for the two cameras, which is needed to obtain expected values of the white reference panel with an accuracy $\pm 3 \%$. However, it should be take into account that those formulas are specific to the researched camera models, lenses used and specific cameras settings. The parameters of these may vary considerably between different models of the same camera and such experiments should be conducted for each model separately. The purpose of this article is to present a methodology for determining these parameters in laboratory conditions.

The obtained accuracy of $\pm 3 \%$ can be further increased by additional measurements in both laboratory and field conditions. The research team from the Department of Remote Sensing and Photogrammetry from the Military University of Technology in Warsaw plan to continue their work on this matter with the hypothesis that, in stable lighting conditions, it is possible to obtain accuracies of around $\pm 1 \%$.

\section{Acknowledgements}

The presented article is part of research work carried out in the "Innovative remote sensing system for the monitoring of pollutants in rivers, offshore waters and flooded areas" project- PBS1/B9/8/2012 financed by the polish National Centre for Research and Development NCBiR.

\section{References}

[1] Kirkbride, K. P. 2000. Spectroscopy: Basic Principles, Encyclopedia of forensic sciences, 194-191.

[2] Von Kauzmann, W. 1957. Quantum Chemistry. An Introduction. Academic Press Inc., New York 1957. Chapter 15

[3] Hollas, J. M. 2004. Modern Spectroscopy, $4^{\text {th }}$ edition, 8-1; 60-40.

[4] Kokaly, R. F.; Despainb, D.G.; Clarka, R. N.; Livoa, K. E. 2001.Mapping vegetation in Yellowstone National Park using spectral feature analysis of AVIRIS data, Remote Sensing of Environment 84(3): 437-456. http://dx.doi.org/10.1016/S0034-4257(02)00133-5

[5] Cheng, Y.-B.; Zarco-Tejada, P. J.; Riaño, D.; Rueda, C. A. 2006. Estimating vegetation water content with hyperspectral data for different canopy scenarios: Relationships between AVIRIS and MODIS indexes, Remote Sensing of Environment 105(4): 354-366. http://dx.doi.org/10.1016/j.rse.2006.07.005

[6] Ruiz-Verdú, A.; Domínguez-Gómez, J.; Peña-Martínez, R. 2009. Use of CHRIS for monitoring water quality in Rosario Reservoir, 3rd ESA CHRIS/Proba Workshop.

[7] Guanter, L.; Alonso, L.; Moreno, J. 2005. First Results From the PROBA/CHRIS Hyperspectral/Multiangular Satellite System Over Land and Water Targets, Ieee Geoscience And Remote Sensing Letters 2(3): 250-254. http://dx.doi.org/10.1109/LGRS.2005.851542

[8] Guanter, L.; Ruiz-Verdú, A.; Odermatt, D.; Giardino, C.; Simis, S.; Estellés, V.; Heege, T.; Antonio Domínguez-Gómez, J.; Moreno, J. 2009. Atmospheric correction of ENVISAT/MERIS data over inland waters: Validation for European lakes, Remote Sensing of Environment 2009, RSE07524

[9] Dębski, W.; Walczykowski, P. 2008. Acquiring reflection coefficients using hyperspectral video imagery, XXIth ISPRS Congress, Beijing China

[10] Han, L. 1997. Spectral reflectance with varying suspended sediment concentration in clear and algae- laden waters, Photogrammetric Engineering \& Remote Sensing 63(6): 701-705.

[11] Scientific brochure Xeva-1.7-320, available at: http://www.xenics.com/documents/XB-003_04_Xeva-1.7-320_Scientific_LowRes.pdf

[12] Scientific brouchure XS-1.7-320, available at: http://www.xenics.com/documents/XB-001_03_XS-1.7-320_Scientific_LowRes.pdf

[13] Serway, R. A.; Jewett, J. W. 2014. Physics for Scientists and Engineers with Modern Physics, $\overline{9}^{\text {th }}$ edition. 